Voix et Images

voixetimages

\title{
D’un animateur littéraire (esquisse historique)
}

\section{Réjean Robidoux}

Volume 7, numéro 1, automne 1981

\section{Adrien Thério}

URI : https://id.erudit.org/iderudit/200301ar

DOI : https://doi.org/10.7202/200301ar

Aller au sommaire du numéro

\section{Éditeur(s)}

Les Presses de l'Université du Québec

ISSN

0318-9201 (imprimé)

1705-933X (numérique)

Découvrir la revue

Citer cet article

Robidoux, R. (1981). D’un animateur littéraire (esquisse historique). Voix et Images, 7(1), 27-34. https://doi.org/10.7202/200301ar d'utilisation que vous pouvez consulter en ligne.

https://apropos.erudit.org/fr/usagers/politique-dutilisation/ 


\title{
D'un animateur littéraire (esquisse historique)
}

\author{
par Réjean Robidoux
}

À l'époque lointaine où je cherchais un sujet de recherche en littérature canadienne - ainsi qu'on disait alors - mon futur directeur de mémoire, féru d'histoire littéraire et qui, en explorant le passé avait le souci de promouvoir la vie présente, m'avait suggéré de tirer au clair le cas de "Casgrain, animateur littéraire ". Engagé dans mon entreprise en toute innocence, je ne fus pas long à découvrir, derrière une jolie façade, certains côtés suspects de mon héros qui me firent très tôt vouloir dissiper les illusions d'une légende au lieu d'en fonder précisément la vérité et d'en proclamer le message. Mais l'expérience en négatif de l'animateur littéraire autour de $1860 \mathrm{~m}$ 'a tout de même préparé à reconnaître d'emblée le modèle authentique, lorsqu'il me serait donné de le rencontrer un siècle plus tard. Ce n'est pas d'abord un grand seigneur, mais un modeste tâcheron, foncièrement généreux, au sens étymologique du terme ("de bonne race») et ("le cœur sur la main») selon l'acception courante. Son ouverture ne laisse aucune place au dogmatisme ou au dirigisme idéologique, car plutôt qu'un système rigide c'est l'idéal sans limite qui l'inspire et qu'il veut promouvoir. Il est certes bien pourvu de l'esprit de combat, mais sa polémique s'exerce essentiellement dans sa lutte pour la vie, et son acharnement exemplaire n'est dépassé que par son abnégation. Témoin engagé, c'est exactement un "martyr", puisque, aussi bien, la passion de dévouement suppose une véritable «dévotion» pour une cause. Il pourrait bien en effet finir par y laisser sa peau, car c'est un homme de foi, c'est quelqu'un qui y croit.

J'ai l'air parti dans le dithyrambe, et pourtant c'est d'un être réel que je parle, et plutôt humble, contesté sourdement par d'aucuns - rares mais tenaces et dotés d'une redoutable force d'inertie - estimé à sa juste valeur par les autres, le commun des mortels, qui le reconnaîtra à son mérite. 
Son intérêt s'est à coup sûr très tôt identifié. «Dois-je quêter ma nourriture terrestre en littérature française ou en littérature canadienne-française? » fera-t-il écrire un jour à l'un des personnages de ses romans ${ }^{1}$. II ne semble pas, quant à lui, avoir longuement hésité dans son choix. En un temps où ne comptait censément que la littérature française, il avait au moins choisi pour sujet de recherche un auteur pour qui le Canada était véritablement devenu une terre d'adoption: scrutant, pour sa thèse de maîtrise en 1951, «l'aspect canadien dans l'ceuvre de Marie Lefranc», il traitait un sujet dont il est loisible de penser qu'il nous appartient, tout autant en tout cas que celui de la très québécoise - et mythologique - Maria Chapdelaine. Et si quelque doute là-dessus malgré tout demeure, l'équivoque sera levée, au stade du doctorat, l'année suivante, avec une thèse sur «Jules Fournier, journaliste de combat».

Néanmoins, ses diplômes obtenus, le jeune professeur non seulement fera ses premières armes à l'étranger, dans des institutions américaines, mais, au fait, il n'enseignera jamais au Québec, en plus de vingt-cinq ans de carrière; et ce n'est pas le moindre paradoxe que ce soit tout de même là que s'exercera son dynamisme d'animateur littéraire. Il partira de loin, en vérité, et procédera sans programme défini, mais, au bout du compte, tout aura servi sa "mission" (que, en bon prophète, je lui reconnais après coup). Je pense, bien entendu, à son œuvre de création, inaugurée en 1953 avec la publication des Brèves Années et qui, de romans en contes et en pièces de théâtre, ne s'est jamais interrompue: on peut très bien la voir, sous l'angle de la stimulation collective, comme le bon exemple qu'on donne afin d'être suivi. Mais, dans un autre ordre de réalité, je songe également à cette brochure de 20 pages, French irregular Verbs/Verbes irréguliers français, sorte de Bescherelle du pauvre, qui, depuis sa parution en 1955 jusqu'aujourd'huı, en 10 éditions distinctes et des tirages totalisant les 186000 , s'est trouvée, comme l'une parmi d'autres sources de revenus, participer d'office aux accomplissements.

C'est en 1957 que l'animateur littéraire a assumé effectivement son rôle, en publiant un premier choix de textes de Jules Fournier; il allait ensuite, en 1965, présenter Mon encrier (enrichi de quelques articles additionnels) du même auteur. La confection d'anthologie vise toujours un but pratique, en rendant accessibles, au moyen d'un groupement significatif et, souvent, de commentaires, des textes qui peuvent alors connaître une utilisation pédagogique importante, sortir de l'ombre et rayonner. L'animateur littéraire est toujours, d'une façon ou d'une autre, un diffuseur de textes. C'est là une partie obligée de sa tâche. II n'est donc pas surprenant de retrouver, dans le cas que j'examine, les étapes suivantes qui jalonnent une carrière jusqu'à ce jour: publication de Conteurs canadiens-français; époque contemporaine, anthologie, en 1965 (2e édition augmentée, en 1970); L'Humour au Canada français. anthologie, en 1968; Témoins du monde français (en collaboration avec James Burk, en 1968 (réédition en 1977); et deux projets en voie de réalisation: L'Éloquence au XIXe siècle et Conteurs québécois 1900-1935.

Et l'on en vient un jour (par quelle suite de hasards calculés?) à tenter 
la grande aventure: la mise en train d'un périodique tout entier consacré aux lettres. En l'occurrence, ce sera Livres et Auteurs canadiens, pour "aider à la diffusion du livre canadien, faire connaître nos auteurs, inviter à les lire ${ }^{2}$. Depuis 1961 que cette revue existe, c'est peu de dire qu'elle est devenue - très vite - un instrument indispensable pour la recherche rétrospective, mais tout autant, je crois, pour la promotion, pour l'orientation, pour la lancée prospective de la littérature. Vous investissez dans cette entreprise je ne sais combien d'argent: il serait sans doute plus juste de dire que vous y engloutissez chaque fois quelques centaines, puis quelques milliers de dollars. Car vous opérez à perte; si, au bout de quelques années, après d'infinies et toujours mortifiantes démarches, vous réussissez à obtenir d'un Conseil des arts et/ou d'un Ministère des affaires culturelles une aide (légitime) bien appréciée, celle-ci "ne [v]ous a pas encore permis de boucler [v]otre budget " courant ${ }^{3}$. Mais vous tiendrez bon, contre vents et marées.

Dès le départ, vous avez sollicité des collaborations «bénévoles» (en fait, dans tous vos projets jusqu'à présent, vous ne cesserez de lutter afin de rémunérer chacun - fût-ce symboliquement - pour les services rendus, au point même que vous serez à peu près le seul à travailler sans compensation: vous baillerez plutôt les fonds, tout en vous improvisant homme à tout faire, vous réservant surtout la difficile besogne de la mise en page - métier peu à peu acquis en quelque sorte à tâtons). La première année, en comptant à la fois votre équipe de directeurs de sections et les auteurs d'articles, vous aviez déjà réuni quelque cinquante collaborateurs; vous ferez état d'une soixantaine. dans le numéro de 1965.

Outre le bilan de chaque année, composé de comptes rendus plus ou moins développés, la revue a adopté la politique de faire paraître dès le second numéro quelque étude sur des sujets plus étendus; cela a pris une telle importance, avec le temps, que ce type d'articles allait atteindre la dizaine dans chaque numéro, au début des années 70 .

En 1966, après cinq ans, la revue modifie son format, et le directeur, en guise de caution morale, s'adjoint un conseil d'universitaires. Puis, par souci d'adaptation à l'usage changeant, Livres et Auteurs canadiens s'appelle Livres et Auteurs québécois, à partir de 1969. C'est une façon en évoluant, de rester le miroir fidèle de la réalité littéraire. En vérité, Livres et Auteurs canadiens ou québécois, affaire personnelle d'un animateur entreprenant, mais, en son genre, véritable institution nationale - au sens où on l'aurait entendu, du temps de Crémazie et de Casgrain -, authentiquement autochtone, a toujours arboré franchement ses couleurs. II n'est donc pas surprenant de relever, parmi les éditoriaux annuels qui tentent, entre autres et à leur manière, de définir notre identité spécifique, des titres comme "Les Français et nous " (1966) et "La lumière nous viendrait-elle de France?" (1971), qui cherchent à nous situer sur la mappemonde. Quant à localiser aussi la revue sur la carte critique, le directeur le fait avec aplomb et une verve certaine (I'article en dialogue lui réussit), dans l'éditorial de 1972 , le dernier de son règne. 
Non pas que Livres et Auteurs disparaisse à cette heure, mais il aura changé de mains en 1973. Déjà, à l'occasion du dixième numéro, en 1970, faisant le point dans un article qui devait par ailleurs se révéler prophétique, l'animateur-fondateur-directeur avait déclaré: «Je ne tiens pas à garder la direction de ce magazine toute ma vie, mais je souhaite que, même entre d'autres mains, il puisse fêter plus tard des anniversaires plus marquants ${ }^{4}$." En fait on pense déjà à quelque chose de différent, mais il faudra du temps pour que l'idée arrive au point de maturité (et, sans doute, aussi pour amasser les fonds nécessaires à un nouveau départ). Toujours est-il que Livres et Auteurs sera cédé, armes et biens, à une équipe de l'Université Laval en 1973.

Entre-temps, l'infatigable avait posé d'autres gestes significatifs dont il faut parler. Je mentionne pour mémoire qu'il ressuscite en mars 1971, sous le titre de Co-Incidences, la petite revue «de création et de réflexion sur la création " des étudiants du Département des lettres françaises de l'Université d'Ottawa, qui, sous la gouverne dynamique et souple du ci-devant fondateur de Livres et Auteurs canadiens/québécois, allait produire dix numéros jusqu'en janvier-février 1974, pour continuer ensuite, telle quelle, sous une autre direction, puis redevenir Incidences (créature, cette fois, des professeurs), en sa plus récente métamorphose.

J'insisterai davantage sur l'apparition dans le paysage littéraire des éditions Jumonville en 1964, parce que cela, entre autres, illustre excellemment un certain type d'épreuves que peut avoir à supporter le promoteur le plus zélé de la littérature. Disons d'abord que j'ai longtemps cru que le choix du nom de cette maison avait pu déceler à l'origine quelque intention polémique (historico-politique), en référence à l' "assassinat » du sieur de Jumonville aux mains de la troupe commandée par George Washington dans I'Ohio en $1754^{5}$. Or, vérification faite récemment auprès du responsable-fondateur des éditions, celui-ci ignorait l'histoire de Jumonville (et l'ignorerait encore si je ne la lui avais sommairement racontée). Les sonorités du nom lui plaisaient seulement, et il y aurait même eu, en l'occurrence, un certain jeu de confusion avec le nom de Bougainville (qu'il préférerait même). $O$ candeur d'âme! Ce que c'est que d'avoir vécu heureux au Kentucky, en Indiana, à Toronto et à Kingston, (à l'ombre du fort Frontenac)!

Quoi qu'il en soit, les éditions Jumonville, qui fournirent un port d'attache et une raison sociale à Livres et Auteurs puis, le moment venu, à un autre périodique, cristallisent un phénomène particulier que j'appellerai le syndrome du parent pauvre et qui n'a pas cessé de caractériser l'homme dont je parle. Il lui est arrivé mainte fois d'essuyer un refus quand il cherchait à publier un manuscrit. Ou bien il n'était pas satisfait de la façon dont l'éditeur professionnel diffusait ses livres (ou les laissait sous le boisseau) ${ }^{6}$. C'est crânement afin de faire ce qu'il voulait, comme il l'entendait, qu'il a fondé Jumonville, et il n'y aurait fait paraître que Les Fous $d^{\prime}$ amour (au préalable, négligé, «refusé» par Le Jour de Jacques Hébert ${ }^{7}$ ) et La Colère du père (écarté par Stanké: «bien que fort intéressant, un texte de cette nature ne cadre pas tout à fait avec nos 
projets immédiats ${ }^{8}$ ), c'eût été bien fait. Mais il $y$ a aussi réédité Ceux $d u$ Chemin Taché et publié C'est ici que le monde a commencé ainsi qu'une poignée de ses livres, sans doute indésirables ailleurs, dont ce "Journal littéraire 1973-1974", Des choses à dire, qui assure pour lui le relais de la parole libre et publique, entre Livres et Auteurs quitté et ce qui va bientôt suivre.

C'est notamment dans l'enchaînement de cette chronique Des choses à dire, que revient dans divers modes (et pour des motifs patents) sa complainte du mal-aimé. Vis-à-vis des establishments, quand on est qui on est, (persona non grata) vous êtes décidément marqué. Du côté de la critique d'abord, l' "officielle» surtout de certains journaux (Le Devoir...), on attendrait parfois un signe, fût-il d'hostilité. Est-il attitude plus frustrante qu'un silence systématique? - Vous êtes, Monsieur, inexistant... Du côté des organismes subventionnaires, Conseil(s) des arts et (bientôt) de recherches en sciences humaines, ou Ministère des affaires culturelles, quand ce n'est pas la politique du compte-gouttes qui prévaut, ce sera le refus (comme en ce début de 1981 au CRSHC, pour un projet de travail libre) ou c'étaient des retards angoissants:

«Quand j'étais directeur de Livres et Auteurs québécois, j'ai attendu moi aussi, je me suis rongé les sangs pendant des mois et des mois, me demandant si les quelques mille dollars que j'avais réclamés, je ne serais pas obligé de les sortir de ma poche et de fermer ma business ensuite. II ne faut pas se surprendre, l'administration des Affaires culturelles, elle est comme ça, elle fait attendre les gens, pour le plaisir de la chose, on dirait. À la fin, elle finit par envoyer un chèque mais seulement quand elle est sûre que ceux qui le recevront ont déjà fait une crise cardiaque en croyant sincèrement qu'ils allaient être obligés de fermer leur porte ${ }^{9}$."

- Seigneur, délivrez-le de ses amis... "Tous ces gens qui me veulent du bien et passent quand même leur temps à me donner des coups de pied au cul finiront peut-être par m'avoir un jour. Pour le moment, je crois encore que c'est moi qui les aurai et c'est ce qui me permet de continuer à travailler ${ }^{10}$.»

II est évident, en cette année 1975, qu'il ne tient pas en place. L'animateur se prépare derechef à appareiller. L'idée remonte loin : l'éditorial de Livres et auteurs 1964 en faisait déjà état '" ; celui de 1970 allait jusqu'à mentionner un titre: Lettres québécoises ${ }^{12}$. Des choses à dire y revient à nouveau, en termes non seulement de souhaits mais de réalisation dans un avenir prochain ${ }^{13}$. La revue sort enfin en mars 1976 . C'est un magazine trimestriel qui se donne pour mission d'être le «témoin de la littérature en marche ${ }^{14}$. En se vouant expressément à l' «actualité littéraire québécoise ${ }^{15}$ \%, la revue occupe d'office un territoire encore inexploité par les périodiques existants ${ }^{16}$ : elle a du reste, dès le second numéro, tenu à faire le point pour qu'on sache «où situer Les Lettres québécoises ${ }^{17}$ " par rapport aux autres, et notamment à côté de Livres et Auteurs qui continue de paraître.

$\mathrm{Si}$, dans cette aventure, un seul homme avance les fonds et assume tous les risques financiers, se réservant la sale besogne alors qu'il rétribue chacun 
pour toute page écrite, c'est, dès le départ, une équipe d'une quinzaine de collaborateurs qui se partage la couverture des quelque dix ou douze rubriques (roman, poésie, théâtre qu'on écrit ou qu'on joue, essai, etc.) de l'actualité. Le directeur (homme à tout faire) s'adjoindra un "comité de régie» (véritable I on peut me croire) à partir du cinquième numéro (février 1977).

Soutenir matériellement (à bout de bras!), fournir aux autres un instrument d'expression vivante, c'est faire œuvre de mécène. La tâche d'animateur littéraire consiste plus spécifiquement dans la lancée et dans la promotion des idées, coïncidant d'ailleurs avec une action concrète. L'homme de Lettres québécoises a deux tribunes dans sa revue: l'éditorial et une chronique «Des choses à dire», et je trouve qu'il s'y manifeste à son meilleur, avec aplomb, avec allant et (quoi qu'on dise) avec style. La manière journalistique sied décidément à merveille à cette plume. Que ce soit sous la forme de la lettre ouverte ${ }^{18}$, du commentaire parodique ${ }^{19}$, d'une sortie polémique ${ }^{20}$, voire d'une étude ${ }^{21}$, ce qui lui réussit le mieux c'est un style direct, un peu «parlé", familier, offrant toutes les possibilités de coups de gueule...

Après «cinq années difficiles ${ }^{22}$, Lettres québécoises a fait ses preuves, nul ne le conteste. La revue n'a cessé de progresser en qualité et dans la faveur publique. À ce stade, elle devrait pouvoir songer à paraître plus souvent, comme ses lecteurs le souhaitent et le lui disent: "L'idéal, ce serait de publier dix numéros par année ${ }^{23}$. Pourtant le numéro 21 (printemps 81) qui vient de sortir, si considérable qu'il soit (72 pages), l'est moins que le précédent (108 pages) et ne devait pas en principe dépasser 48 pages, après avoir failli ne pas paraître du tout. Lettres québécoises (c'est-à-dire, en prose d'affaires, son principal pourvoyer de capital) lutte pour sa survie. Ce n'est pas là, ironiquement, une grosse surprise. La prévision avait été faite au départ, voilà cinq ans, puisque le maître d'œuvre écrivait lui-même dans le premier éditorial : "Je sais, sans faire d'enquête, qu'une revue comme celle-ci n'est pas rentable et qu'elle devra vivre de subventions ${ }^{24}$." C'est l'évidence même: toutes les publications de cette sorte ne subsistent ici que sur ce que leur octroient les mécènes publics (Ministères et autres Conseils). Mais il ne suffit pas de solliciter pour recevoir, et du reste le don ne correspond pas toujours au besoin même sobre et honnêtement déclaré. Lettres québécoises (je veux toujours dire: son responsable) a entrepris tractations et démarches auprès du Conseil des arts du Canada dès l'été 1976, après la sortie du deuxième numéro, afin d'être en posture de toucher une aide à partir du quatrième (en novembre 1976), comme cela se pratique selon les règlements établis. Je voudrais le crier sur les toits, parce que, depuis le début et tout au long des escarmouches, je me suis trouvé (au point d'y être moi-même impliqué) le témoin des demandes, des refus, (des colères, des silences) des promesses (et des blocages). Quand une première subvention de $\$ 10000$ a tout de même fini par être accordé, l'on était déjà à l'automne 1978, la revue avait deux ans et demi d'existence (héroïque) et onze numéros (non pas seulement trois) étaient parus. Depuis lors le Conseil a renouvelé une fois (à l'automne 1979) ce premier montant (tel quel, malgré les réclamations insistantes), puis, une seconde fois (à l'automne 1980), il l'a 
augmenté de la rallonge espérée de longs mois l'année précédente et qui s'était, à la fin, dérobée. De sorte que la revue reçoit cette année $\$ 15000$. quand il lui en faudrait le double.

Que va-t-il à présent arriver? Dans l'éditorial tout frais sorti de presse, on peut lire cette phrase: [ ...] «nous savons qu'il nous faudra faire appel à une autre source de crédit avant l'arrivée des subventions pour l'année 81 $822^{25}$ \%. Cela signifie en langage franc qu'il faudra faire un emprunt (remboursable à quel taux d'intérêt?) dans une banque. Et qui s'engagera? qui signera? je le demande. - Toujours lui, qui n'inscrira même pas son véritable patronyme (i) l'a laissé sans partage à son grand cousin Yves). II fera plutôt, à sa façon coutumière, le brave type (pas riche) qui écrit son nom au son et qui ne se fait pas un mérite spécial de s'appeler ADRIEN THÉRIO.

1. Un paien chez les pingouins, Mantréal, CLF, 1970, p. 115

2. Livres et Auteurs canadiens 1961, p. 4.

3. Livres et Auteurs canadiens 1965, p. 3.

4. Livres et Auteurs québécois 1970, p. 8.

5. Voir: Marcel Trudel, "L'affaire Jumonville», dans Revue d"histoire de l'Amérique française, VI, $n^{\circ} 3$ (décembre 1952), p. 331-373.

6. Voir l'échange de lettres avec Pierre Tisseyre, dans Des choses à dire, Montréal, Éditions Jumonville, 1975, p. 104-114 et 117-120.

7. Ibid., p. 129.

8. Ibid., p. 129-135.

9. Ibid., p. 157-158.

10. Ibid., p. $171-172$.

11. Livres et Auteurs canadiens 1964, p. 4

12. Livres et Auteurs québécois 1970, p. 7. "J'avais complètement oublié cet éditorial quand j'ai vu Les Lettres québécoises en marche, dira-t-il en 1978. C'est, il y a quelques mois seulement, que par hasard, j'ai relu ce discours. Si je le reprends aujourd'hui, c'est pour bien montrer que je $n$ 'ai pas changé d'idée quant à l'essentiel. Et on devrait savoir maintenant que ces deux revues (Livres et Auteurs et Lettres québécoises) n'ont rien de très ressemblant. Si le programme d'aujourd'hui diffère assez de celui que je proposais il y a 8 ans, c'est que, dans mon esprit, la revue que je fondais en 1976 se devait de parler surtout des écrivains qui publient maintenant. Et si j'ai décidé un jour de me lancer dans cette aventure, c'est que, inconsciemment, j'ai dû m'imaginer que six années d'attente, c'était assez. Mais, en fait, je n'en sais trop rien.» (Lettres québécoises, $n^{\circ} 10$, avril 1978, p. 4.)

13. Des choses à dire, p. 120-166.

14. Les Lettres québécoises, $n^{\circ} 1$, mars $1976, p .3$. L'article (les) restera dans le titre jusqu'au numéro 10 . Il disparaîtra au numéro 11, en même temps qu'aura changé (couleurs mélangées) le style de présentation de la couverture.

15. lbid., (et sur les numéros suivants), page de couverture.

16. Il n'entre pas dans mon propos de vérifier ici la rumeur qui a couru à l'époque et qui voulait qu'un autre "groupe", doté de moyens moins artisanaux, lance justement une revue d'actualités littéraires. La parution de Lettres québécoises, qui n'avait pourtant rien d'improvisé, se serait ainsi trouvée devancer - et contrecarrer - certains desseins. 
17. Les Lettres québécoises, $n^{\circ} 2$, mai 1976, p. 2.

18. Les Lettres québécoises, $n^{\circ} 4$, novembre 1976, p. 4-6; «Lettre ouverte au Ministre des Affaires culturelles".

19. «Un grand garçon qui nous veut du bien*, dans Lettres québécoises, $n^{\circ} 5$, février 1977, . 40-41.

20. «Conseil des arts du Canada. Aide aux périodiques», dans Lettres québécoises, $n^{\circ}$ 7, août-septembre 1977, p. 4-5; "Les grands projets d'édition du Conseil des Arts et le Canada anglais», dans Lettres québécoises, no 12, novembre 1978, p. 4-5; «Nos écrivains à l'écran: Pourquoi Radio-Canada et Radio-Québec refusentelles de bouger?", dans Lettres québécoises, $n^{\circ} 14$, avril-mai 1979, p. 4.

21. "Chronique des années perdues (Leméac) de Guy Frégault", dans Lettres québécoises, $\mathrm{n}^{\circ} 7$, août-septembre 1977, p. 52-54.

22. Lettres québécoises, $n^{\circ} 20$, hiver 1980-1981, p. 9.

23. Lettres quebecoises, $n^{\circ} 21$, printemps 1981, p. 9.

24. Les Lettres québécoises, $n^{\circ} 1$, mars 1976, p. 3.

25. Lettres québécoises, $n^{\circ} 21$, printemps 1981, p. 9. 